\title{
THE STRUCTURE OF TORSION ABELIAN GROUPS GIVEN BY PRESENTATIONS ${ }^{1}$
}

\author{
BY PETER CRAWLEY AND ALFRED W. HALES
}

Communicated by R. S. Pierce, March 1, 1968

Let $F_{X}$ denote the free abelian group freely generated by the set $X$, and let $R$ be a subset of $F_{X}$. With $[R]$ denoting the subgroup of $F_{X}$ generated by $R$, set

$$
G(X, R)=F_{X} /[R],
$$

i.e., $G(X, R)$ is that abelian group generated by $X$ and subject only to the relations

$$
r=0 \quad \text { all } r \in R .
$$

If each of the elements in $R$ involves only one generator in $X$, then $G(X, R)$ is a direct sum of cyclic groups. On the other hand, if $G$ is any abelian group, then $G \cong G(X, R)$, where each element in $R$ involves at most three generators in $X$; indeed this isomorphism results if we take $X=G$ and $R$ equal to the set of all elements in $F_{G}$ of the form $x+y-z$, where $z=x+y$ in $G$.

Our purpose here is to investigate the structure of the group $G(X, R)$ in the intermediate case when each of the elements of $R$ involves at most two generators, and $G(X, R)$ is a torsion group. We can evidently restrict our attention to $p$-groups, and in this case it is easily seen that $G(X, R) \cong G\left(X^{\prime}, R^{\prime}\right)$, where each element in $R^{\prime}$ is of one of the forms

$$
p^{n} x \text { or } p^{n} x-y \text {. }
$$

This leads us to the following definition. Let $X$ be a set, $V$ be a subset of the set of ordered pairs $\langle x, y\rangle$ with $x, y \in X, u$ be a map of $X$ to the nonnegative integers, and $v$ be a map of $V$ to the nonnegative integers. By $G(X, V, u, v)$ we mean that abelian group generated by $X$ and subject only to the relations

$$
\begin{array}{ll}
p^{u(x)} x=0 & \text { all } x \in X, \\
p^{v(x, y)} x=y & \text { all }\langle x, y\rangle \in V .
\end{array}
$$

We say that an abelian $p$-group $G$ is a $T$-group if $G \cong G(X, V, u, v)$ for some $\langle X, V, u, v\rangle$.

\footnotetext{
1 This work was supported in part by NSF Grants GP 7252 and GP 5497.
} 
One property of $T$-groups is clear: the direct sum of a family of $T$ groups is again a $T$-group. Every divisible $p$-group is certainly a $T$ group, and the reduced part of a $T$-group is again a $T$-group.

Before stating our main results concerning these groups, let us recall a few basic definitions. Let $G$ be any reduced abelian $p$-group. Define the subgroups $p^{\alpha} G$ for each ordinal $\alpha$ as usual by the rules: $p^{0} G=G ; p^{\alpha} G=\left\{p x \mid x \in p^{\alpha-1} G\right\}$ if $\alpha-1$ exists; $p^{\alpha} G=\bigcap_{\beta<\alpha} p^{\beta} G$ if $\alpha$ is a limit ordinal. Since $G$ is reduced, there is a first ordinal $\lambda$, called the length of $G$, such that $p^{\lambda} G=0$. For each ordinal $\alpha$ we set

$$
f_{G}(\alpha)=\operatorname{rank} p^{\alpha} G \cap G[p] / p^{\alpha+1} G \cap G[p],
$$

where $G[p]=\{x \in G \mid p x=0\}$, and we call the cardinal number $f_{G}(\alpha)$ the $\alpha$ th Ulm invariant of $G$. Finally we let $\omega$ denote the first infinite ordinal and $\Omega$ denote the first uncountable ordinal.

The description of $T$-groups is now accomplished by the following theorems.

(A) If $G$ and $H$ are reduced $T$-groups, then $G$ and $H$ are isomorphic if and only if $f_{G}(\alpha)=f_{H}(\alpha)$ for each ordinal $\alpha$.

(B) Let $f$ be a map of an ordinal $\lambda$ to a set of cardinal numbers. Then there exists a reduced $T$-group $G$ of length $\lambda$ such that $f_{G}(\alpha)=f(\alpha)$ for each $\alpha<\lambda$, if and only if $f$ satisfies the following conditions:

(i) $\lambda=\sup \{\alpha+1 \mid f(\alpha) \neq 0\}$;

(ii) if $\alpha$ is a limit ordinal such that $\alpha+\omega<\lambda$, and $0 \leqq \eta<\omega$, then

$$
\sum_{\alpha+\eta \leq \beta<\alpha+\omega} f(\beta) \geqq \sum_{\alpha+\omega \leq \beta<\lambda} f(\beta) .
$$

(C) A reduced p-group $G$ is a direct sum of countable groups if and only if $G$ is a $T$-group of length at most $\Omega$.

When specialized to countable $p$-groups, (A) and (C), of course, reduce to Ulm's Theorem, and in the case of direct sums of countable groups they reduce to the theorem of Kolettis [2]. Our results are not independent of Ulm's Theorem, however, since it is used to establish (C). The proofs of (A), (B) and (C) will appear elsewhere.

Actually $T$-groups have been studied before in a different guise. In [3], Nunke defines a reduced $p$-group $G$ to be totally projective if

$$
p^{\alpha} \operatorname{Ext}\left(G / p^{\alpha} G, A\right)=0
$$

for all ordinals $\alpha$ and every group $A$, and he obtains a number of properties of these groups. Quite recently Hill [1] has announced that two totally projective groups with the same Ulm invariants are isomorphic. Now it is easily verified that if $G$ is a reduced $T$-group and $\alpha$ is 
an ordinal, then both $p^{\alpha} G$ and $G / p^{\alpha} G$ are $T$-groups. Moreover, (A) and (B) yield that a T-group whose length is a limit ordinal is a direct sum of T-groups of smaller length. These last two facts, in conjunction with $[3,2.6]$, imply that every reduced $T$-group is totally projective. On the other hand, if $H$ is a totally projective group, then the function $f_{H}$ necessarily satisfies condition (ii) of (B). Consequently there is a reduced $T$-group $G$ having the same Ulm invariants as $H$, and Hill's theorem guarantees that $G$ and $H$ are isomorphic. Thus $a$ reduced abelian p-group is totally projective if and only if it is a T-group.

The foregoing results further provide a characterization of the class of all reduced $T$-groups in terms of certain natural group-theoretic properties. Let $\mathfrak{K}$ be a class of reduced abelian p-groups. Then $\pi \mathcal{K}$ coincides with the class of all reduced T-groups if and only if $K \mathcal{K}$ has the following properties: (1) $K$ is closed under isomorphism; (2) $K$ is closed under direct sums; (3) if $G \in \Re$ and the length of $G$ is a limit ordinal, then $G$ is a direct sum of groups in $\Re$ of smaller length; (4) for each $p$-group $G$ and each ordinal $\alpha, G \in \mathcal{K}$ if and only if both $G / p^{\alpha} G, p^{\alpha} G \in K$; (5) if an abelian p-group $G$ has no elements of infinite height, i.e., $p^{\omega} G=0$, then $G \in \mathcal{K}$ if and only if $G$ is a direct sum of cyclic groups. Thus the class of all reduced $T$-groups is the smallest class of reduced $p$-groups that has properties (1)-(4) and contains the finite groups.

\section{REFERENCES}

1. P. Hill, Ulm's theorem for totally projective groups, Notices Amer. Math. Soc. 14 (1967), 940.

2. G. Kolettis, Direct sums of countable groups, Duke Math. J. 27 (1960), 111-125.

3. R. Nunke, Homology and direct sums of countable abelian groups, Math. Z. 101 (1967), 182-212.

VANDERBILT UNIVERSITY,

California Institute of Technology and

University of California, Los ANGeles 